\title{
Protocol for harvesting adult human hippocampus
}

\author{
Mathangasinghe $\mathrm{Y}^{1,2 *}$, Handunnetti $\mathrm{SM}^{3}$, Warawitage $\mathrm{DH}^{4}$, Dissanayake $\mathrm{DN}^{5}$ \\ ${ }^{1}$ Neurodegeneration Pathobiology Laboratory, Queensland Brain Institute, University of \\ Queensland, Brisbane, Australia \\ ${ }^{2}$ Department of Anatomy, Faculty of Medicine, University of Colombo \\ ${ }^{3}$ Institute of Biochemistry Molecular Biology and Biotechnology, Faculty of Medicine, \\ University of Colombo \\ ${ }^{4}$ Department of Biochemistry, Faculty of Medicine, University of Colombo \\ ${ }^{5}$ Department of Physiology, Faculty of Medicine, University of Colombo \\ *yasith@anat.cmb.ac.lk
}

Hippocampus has gained much attention recently as a niche of adult neural stem cells, thus a potential therapeutic target in neurodegenerative diseases. Methods of harvesting hippocampi in rodents are described in detail. Here, we aimed to develop a protocol to harvest adult human hippocampi from post-mortem brain specimens. Brains were fixed en-bloc in $10 \%$ paraformaldehyde for 18 hours. Dorsal approach was selected as the best approach after testing ventral and lateral approaches. Five-millimetrethick axial sections were made starting from the level of corpus callosum to expose the lateral ventricles. A 14gauge needle was inserted into the temporal horn of the lateral ventricle and the position of the anterior and posterior ends of the temporal horn was marked on the cortex by piercing the temporal lobe with the needle. An incision was made on the temporal lobe joining the above mentioned two points to open the temporal horn. Hippocampus was visualized and separated from the parahippocampal gyrus by "rolling", starting from its posterior end. The amygdala was dissected with the hippocampus at the anterior end to preserve its connections. Macroscopically, the isolated tissue consisted of entire hippocampus and dentate gyrus. The proposed method of harvesting human hippocampus was repeatable. We plan to validate our protocol by visualizing different regions of the harvested hippocampal tissue after immunostaining.

Acknowledgements:

AHEAD Operation of the Ministry of Higher Education funded by the World Bank. 